\title{
Comparison of antioxidant activity assays in fresh purple waxy corn (Zea mays L.) during grain filling
}

\author{
Jung-Tae Kim ${ }^{1 \dagger}$, III-Min Chung ${ }^{2 \dagger}$, Mi-Jung Kim³ ${ }^{3}$ Jin-Seok Lee', Beom-Young Son ${ }^{3}$, Hwan-Hee Bae ${ }^{3}$, \\ Young Sam Go ${ }^{3}$, Sun-Lim Kim ${ }^{3}$, Seong-Bum Baek ${ }^{3}$, Seung-Hyun Kim² and Gibum Yi ${ }^{4^{*}}$ (D)
}

\begin{abstract}
Purple waxy corn is a good source of antioxidant compounds such as anthocyanins and polyphenols. Promotion of its use requires an appropriate assay to determine antioxidant activity. The aim of this study is to verify compatibility of the antioxidant activity assays by comparing five different assays in daily kernel samples of the fresh purple waxy corn during grain filling. The levels of antioxidants measured by 2,2-diphenyl-1-picrylhydrazyl, ferric reducing antioxidant power, and chemiluminescence showed significant positive correlations with the levels measured by the other assays $(r=0.761-0.893 ; p<0.01)$ and with anthocyanin content $(r=0.798-0.924 ; p<0.01)$. Reducing capacity of $2,2^{\prime}$-azinobis (3-ethylbenzothiazoline-6-sulfonic acid) and total phenolic content also showed significant correlations $(r=0.764$; $p<0.01)$. However, during late grain filling, the levels of antioxidants measured by all methods showed strong correlations with each other $(r>0.699 ; p<0.05)$. The inconsistencies among the measurements are largely dependent on the developmental stage of the corn kernel. The combination of at least two assays is required to ensure reliable antioxidant activity estimates, especially for early grain-filling stages. These results will inform efforts to promote fresh purple corn as a source of antioxidants.
\end{abstract}

Keywords: Anthocyanin, Antioxidant activity, Corn, Grain filling, Phenolics

\section{Introduction}

Purple corn has attracted attention as a source of antioxidant compounds as well as natural dyes [1-3]. These antioxidant compounds, which include phenolics and anthocyanins, are valued for their potential to reduce the risk of a variety of diseases $[4,5]$ due to their anti-inflammatory [6, 7], anti-cancer [8-10], and anti-obesity effects [11-13].

Waxy corn is mostly consumed as a fresh vegetable and its production is increasing, especially in Asian countries $[14,15]$. The antioxidant qualities of corn are established

\footnotetext{
*Correspondence: gibumyi@cnu.ac.kr

†Jung-Tae Kim and III-Min Chung contributed equally to this work

${ }^{4}$ Department of Bio-Environmental Chemistry, College of Agriculture and Life Sciences, Chungnam National University, Daejoen 34134,

Republic of Korea

Full list of author information is available at the end of the article
}

during grain filling and various studies have evaluated the antioxidant activity of diverse phytochemicals in purple corn kernels. For example, Lopez-Martinez et al. [16] reported that differences in free-radical scavenging and reducing activities in purple corn kernel extracts depend on the unique profile of anthocyanins and other phenolic compounds present in each genotype. The seeds and cobs of purple corn reportedly possess excellent antioxidant properties, which might increase the potential for industrial application of these natural pigments [17]. Hu and $\mathrm{Xu}$ observed that black waxy corn contained a high level of anthocyanins and phenolics, together with high antioxidant activity compared with yellow and white corn, which possessed a lower level of anthocyanins, phenolics, and antioxidant activity [14].

Accurate measurement of antioxidant activity is essential to inform the public, medical and nutritional 
experts, and researchers, about the antioxidant activities of foods. Many foods contain a variety of constituents with the potential for synergistic interactions between antioxidant compounds; however, the separation and individual identification of each antioxidant compound is expensive and inefficient; therefore, many studies assay total antioxidant activity [18, 19]. The methods used to assay antioxidant activity in vitro have been classified into three categories: (i) those involving hydrogen atom transfer reactions $(\mathrm{ROOH} \cdot+\mathrm{AH} \rightarrow \mathrm{A} \cdot)$; (ii) those involving electron transfer reactions (M $(n)+\mathrm{e}($ from $\mathrm{AH}) \rightarrow \mathrm{AH} \cdot+\mathrm{M} \quad(n-1)$; and (iii) those with mechanisms involving enzymes, such as superoxide dismutase $[19,20]$. Assays involving hydrogen atom transfer reactions include oxygen radical-absorbance capacity, total radical-trapping antioxidant capacity, and the inhibition of oxygen uptake, linoleic acid oxidation, or low-density lipoprotein oxidation. Assays based on electron transfer reactions include the reducing capacity of 2,2'-azinobis (3-ethylbenzothiazoline-6-sulfonic acid) (ABTS), ferric-reducing antioxidant power (FRAP), scavenging capacity for 2,2-diphenyl-1-picrylhydrazyl (DPPH) free radicals, copper (II) reduction capacity, and total phenolic content (TPC) as estimated using Folin-Ciocalteu reagent. Assay methods that employ other approaches include measurements of the total oxidant scavenging capacity, the degree of inhibition of the Briggs-Rauscher oscillation reaction, and chemiluminescence (CHE) or electrochemiluminescence. Huang et al. [19] suggested that the use of several methods to analyze in vitro antioxidant activity can cover a broad variety of potential antioxidant mechanisms and thus may help to overcome the contradictory results usually obtained by using different antioxidant assays to analyze the same materials [21].

In the previous report, we showed that 'Heukjinjuchal' has the highest level of anthocyanin in kernel among purple cultivars, and it accumulated during grain filling. Since the level of anthocyanin is gradual during the grain filling stage $[22,23]$, it is an appropriate material to check the consistency of different methods in various levels of anthocyanin contents.

To date, various methods have been applied to measuring the antioxidant activity of corn kernels. However, most of these assays used dry kernels and showed different results among many samples tested and across experiments and laboratories. Thus, the aim of this study was to compare the efficiency of DPPH, FRAP, ABTS, CHE, and TPC assays to estimate antioxidant activities and their correlations in fresh purple corn kernels. We could evaluate the antioxidant activity of 'Heukjinjuchal' purple corn kernels using these different methods.

\section{Materials and methods}

\section{Sample preparation}

Maize (Zea mays) 'Heukjinjuchal' is a single-cross hybrid cultivar developed at the National Institute of Crop Science in South Korea [24]. It is one of the dominant cultivars of fresh waxy corn in the South Korean seed market and accumulates anthocyanin in the purple aleurone. Kernels were collected daily starting from 15 days after silking (DAS) to $31 \mathrm{DAS}$, for a total of 17 sampling time points. Samples were collected from the middle of the cob to reduce the variation in color. Harvested kernels were immediately frozen in liquid $\mathrm{N}_{2}$ and stored at $-80{ }^{\circ} \mathrm{C}$ until use. All the following measurements were done within a month from the first sampling time.

\section{Determining the anthocyanin content}

The anthocyanin content was measured as previously described [25]. One gram of corn kernel powder was mixed with $0.1 \mathrm{~N} \mathrm{HCl}$ in methyl alcohol. The mixture was shaken for $30 \mathrm{~min}$ in the dark at room temperature and centrifuged at $4500 \times g$ for $20 \mathrm{~min}$. The absorbance value of the supernatant was measured at $535 \mathrm{~nm}$ with a spectrophotometer (U-3900 Hitachi, Tokyo, Japan). The standard curve was obtained with 1, 10, and $100 \mathrm{mg} \mathrm{g}^{-1}$ cyanidin-3-glucoside (Extrasynthese, Genay, France) and the values were converted according to the standard curve.

\section{DPPH assay}

Kernel extracts of purple corn were assayed using DPPH as described [26]. One gram of kernel powder was extracted with $80 \%$ methyl alcohol for $24 \mathrm{~h}$ at room temperature with stirring. The extracted solution was then filtered through Whatman No. 42 filter paper. Reaction mixtures were prepared by mixing $2.5 \mathrm{~mL}$ DPPH (Sigma Aldrich, MO, USA) solution (0.35 mM DPPH dissolved in $50 \% \mathrm{EtOH}$ ) with $0.2 \mathrm{~mL}$ of each extract. The reaction was incubated for $10 \mathrm{~min}$ at room temperature and changes in DPPH absorbance at $517 \mathrm{~nm}$ were measured with a spectrophotometer (U-3900 Hitachi, Tokyo, Japan). The amounts were calculated as $\mu$ mol Trolox equivalents $\mathrm{g}^{-1}$ dry weight based on a standard curve generated with Trolox solution $(100-1000 \mu \mathrm{M})$.

\section{FRAP assay}

The reducing ability of each sample extract was determined using the FRAP assay [14, 26]. Briefly, FRAP reagent (Sigma Aldrich, MO, USA) was prepared by mixing $300 \mathrm{mM}$ sodium acetate buffer ( $\mathrm{pH} 3.6), 10 \mathrm{mM}$ 2,4,6-tripyridyls-triazine solution in $40 \mathrm{mM} \mathrm{HCl}$, and $20 \mathrm{mM} \mathrm{FeCl}{ }_{3}$ solution at a ratio of 10:1:1 (v/v/v). The FRAP reagent was freshly prepared and warmed in a water bath to $37{ }^{\circ} \mathrm{C}$ prior to use; $0.1 \mathrm{~mL}$ of sample 
extract was mixed with $1.8 \mathrm{~mL}$ FRAP reagent and $3.1 \mathrm{~mL}$ ultrapure water (high-performance liquid chromatography grade). The reaction mixture was incubated at $37^{\circ} \mathrm{C}$ for $30 \mathrm{~min}$ prior to measuring the absorption of the mixture at $593 \mathrm{~nm}$. FRAP values were calculated from a $\mathrm{FeSO}_{4}$ standard curve $(100-1000 \mu \mathrm{M})$ and reported as $\mu$ moles Fe (II) $\mathrm{g}^{-1}$ dry sample weight.

\section{ABTS assay}

The ABTS assay measures the reduction of the radical cation of $\mathrm{ABTS}^{+}$and was carried out as previously described, with slight modifications [14, 26]. Briefly, $\mathrm{ABTS}^{+}$was generated by reacting $7 \mathrm{mM}$ ABTS with $2.45 \mathrm{mM}$ potassium persulfate. The reaction mixture was allowed to stand in the dark at $24^{\circ} \mathrm{C}$ for $16-24 \mathrm{~h}$ and was then used within 2 days. The $\mathrm{ABTS}^{+}$solution was diluted with methanol to an absorbance of $0.700 \pm 0.050$ at $734 \mathrm{~nm}$. All samples were diluted to obtain $20-80 \%$ inhibition of blank absorbance; $50 \mu \mathrm{L}$ of each diluted extract was mixed with $1.9 \mathrm{~mL}$ diluted $\mathrm{ABTS}^{+}$solution. Assays were performed in triplicate and incubated for $6 \mathrm{~min}$ at $24{ }^{\circ} \mathrm{C}$ prior to measuring the absorbance at $734 \mathrm{~nm}$. Trolox solution $(100-1000 \mu \mathrm{M})$ was used as a reference standard. The results were expressed as $\mu$ mol Trolox equivalents $\mathrm{g}^{-1}$ dry weight.

\section{CHE assay}

The CHE assay was performed as described by Kim et al. [27]. Briefly, sample extracts $(10 \mu \mathrm{L})$ were mixed with ARAW-KIT (anti-radical ability of water-soluble substance) from ABCD GmbH (Berlin, Germany). The signal was directly measured using a chemoluminometer (minilum L100, ABCD GmbH, Berlin, Germany) at $37{ }^{\circ} \mathrm{C}$. Ascorbic acid (Sigma Aldrich, MO, USA) was used to make the antioxidant activity standard curve. The values were further converted to ascorbic acid equivalents.

\section{Measurement of total polyphenol contents (TPC)}

Quantitative analysis of TPC was performed using the Folin-Dennis colorimetric method [28]. Briefly, sample extracts $(0.5 \mathrm{~mL})$ were mixed with $5 \mathrm{~mL}$ distilled water and $5 \mathrm{~mL}$ Folin-Ciocalteu phenol reagent in screwtop flasks. After $3 \mathrm{~min}, 2 \mathrm{~mL} 10 \%$ sodium carbonate $\left(\mathrm{Na}_{2} \mathrm{CO}_{3}\right)$ was added and the mixtures were stirred in a shaker chamber at $30{ }^{\circ} \mathrm{C}$ for $1 \mathrm{~h}$. Absorbance of the solutions was measured at $760 \mathrm{~nm}$. A standard curve was obtained using 1-100 ppm gallic acid (Sigma Aldrich, $\mathrm{MO}$, USA) and the results were expressed as $\mu \mathrm{g}$ gallic acid equivalents $\mathrm{g}^{-1}$ dry weight.

\section{Statistical analysis}

The antioxidant activities from the five different assays for 51 samples (3 biological replicates from different ears $\times 17$ time points) were used for principal component analysis (PCA). Auto-scaling and PCA were conducted using MetaboAnalyst 5.0 (https://www.metaboanalyst. ca) [29]. The data obtained from these assays were also analyzed using Excel (Microsoft Office 2016, WA, USA) and SAS software version 9.3 for Windows (SAS Institute Inc., NC, USA). Pearson's correlation was used to compare the measurements of assay methods for purple corn during early grain filling.

\section{Results and discussion}

\section{Measuring the anthocyanin content}

Anthocyanin purple pigments accumulated during grain filling and were first observed at 16 DAS in the middle of a cob; the cobs were fully colored at 30 DAS (Fig. 1A). When anthocyanins were measured using spectrophotometry, the amount continuously increased and reached a maximum of $2.44 \mathrm{mg} \mathrm{g}^{-1}$ fresh weight at 30 DAS (Fig. 1B, Additional file 1: Table S1). The increasing accumulation of anthocyanins was sufficient for correlation analysis, although the amount was larger than that reported previously $[16,30]$. A more precise quantification of anthocyanins using HPLC would be necessary to improve the correlation. Due to the gradual accumulation of anthocyanins, daily samples represent a good source material for investigating correlations among measurements from different antioxidant activity assays.

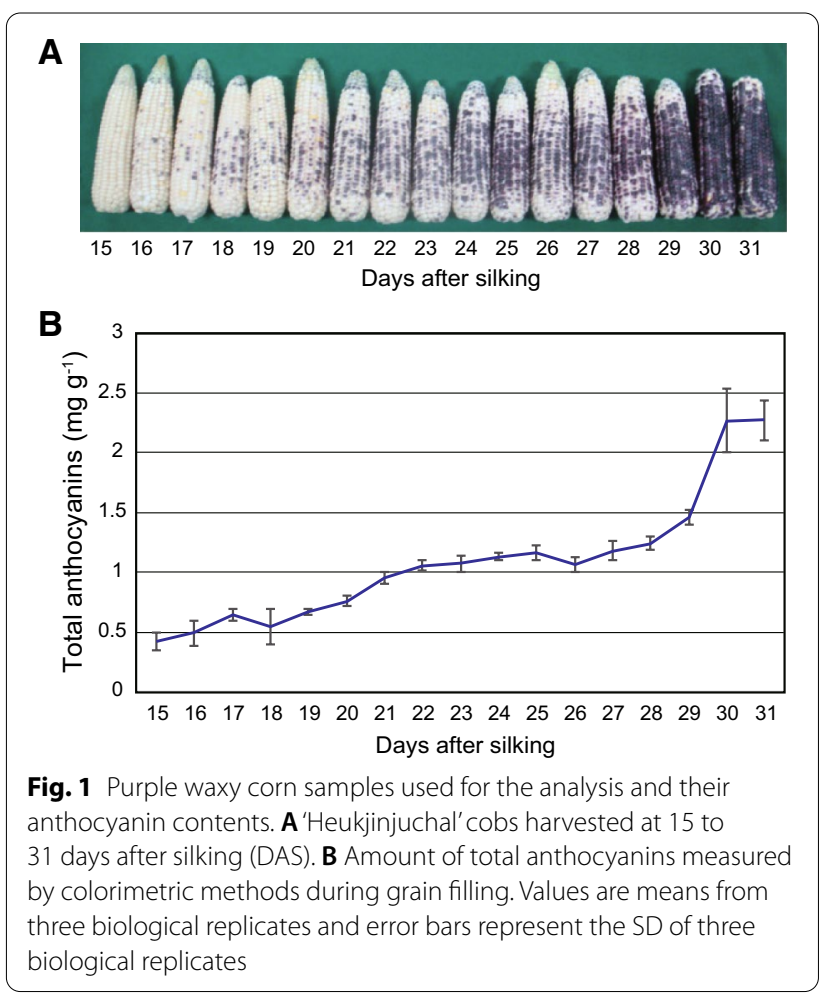




\section{Overview of the sample characteristics based on principal} component analysis

The two components, $\mathrm{PC} 1$ and $\mathrm{PC} 2$, covered $85 \%$ of the total variation among samples (Fig. 2A). The all five antioxidant values were positively loaded to $\mathrm{PC} 1$ with the highest loading of FRAP. CHE and DPPH were positively and consistently loaded to PC2 whereas, TPC and ABTS showed negative loading to PC2 (Fig. 2B). The samples were well arranged according to the DAS. The 23-28 DAS samples were clustered together in the middle of the score plot. Three replicates at the same DAS were closely located, where the 20 DAS samples showed relatively high variation among biological replicates (Fig. 2A).

\section{Measuring antioxidant activity with five different assays}

The antioxidant activity of extracts of 'Heukjinjuchal' purple corn kernels was evaluated during the grain filling period using five different assays. Radical scavenging activity by DPPH tended to increase during grain filling and ranged from 6.45 to $12.90 \%$. The value was lowest at 20 DAS and reached its highest level at 30 DAS. The FRAP assay showed the largest variation among samples, with high standard error among biological replicates.

The ABTS assay reflects the ability to reduce the stable radical $\mathrm{ABTS}^{+}$, relative to the activity of the antioxidant standard, Trolox [31]. The ABTS activity was the highest at 15 DAS and then decreased until 22 DAS when it reached the lowest value. The ABTS activity subsequently increased until 31 DAS, when it reached its second highest value (Fig. 3, Additional file 1: Table S1). The ABTS assay was developed by Miller et al. [32] and its operational simplicity led to its wide adoption for measuring antioxidant activity [19].

Chemiluminescence (CHE) is measurement of the emission light that results from a chemical reaction [33]. The antioxidant activity estimated by the CHE assay generally increased during grain filling: the lowest and the highest values were observed at 16 and 30 DAS, respectively (Fig. 3, Additional file 1: Table S1). The increase at the late grain-filling stages is thought to relate to the increase in anthocyanin content with reduced moisture content in the corn kernel.

The use of the TPC assay to measure the reducing activity of sample extracts reportedly produced a linear correlation between antioxidant activity and the phenolic compound contents [14, 26, 30, 34]. However, information concerning the change in TPC assay signal from fresh corn kernels during grain filling is limited. The TPC was highest at 15 DAS and lowest at 22 DAS (Fig. 3, Additional file 1: Table S1).

A variety of assay methods have been used to determine antioxidant activity and the use of more than one assay has been recommended, as these may produce inconsistent results $[19,20]$. The FRAP, CHE, and DPPH values showed a similar pattern during grain filling, fluctuating between 17 and 27 DAS, increasing from 27 to 30 DAS, and reaching a maximum at 30 DAS. The

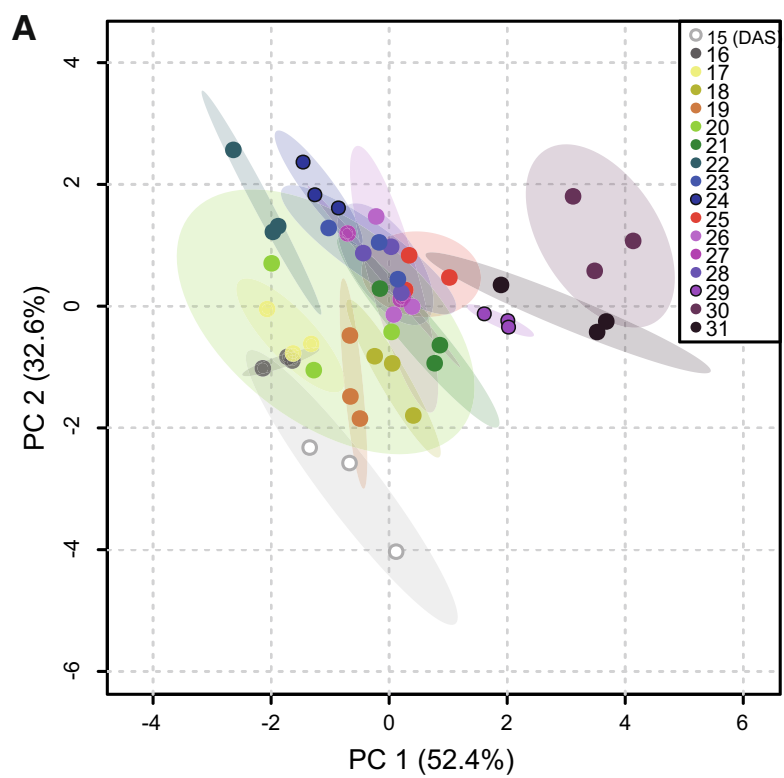

B

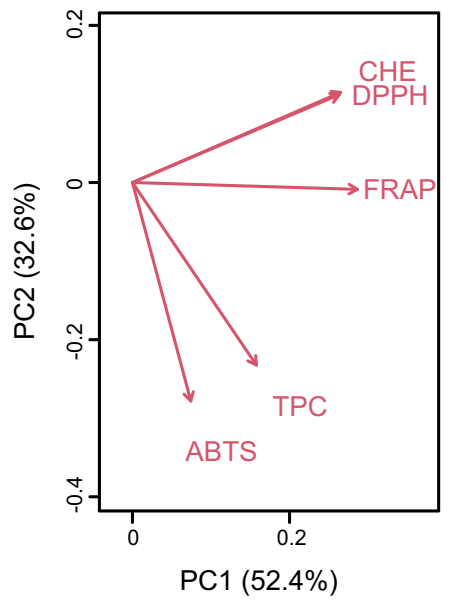

Fig. 2 Principal component analysis of the corn kernel samples during seed maturation based on the five different antioxidant activity measurements. A Score plot showing gradual arrangement of samples according to their days after silking (DAS). B Loading plot of five different antioxidant measuring assays for PC1 and PC2 


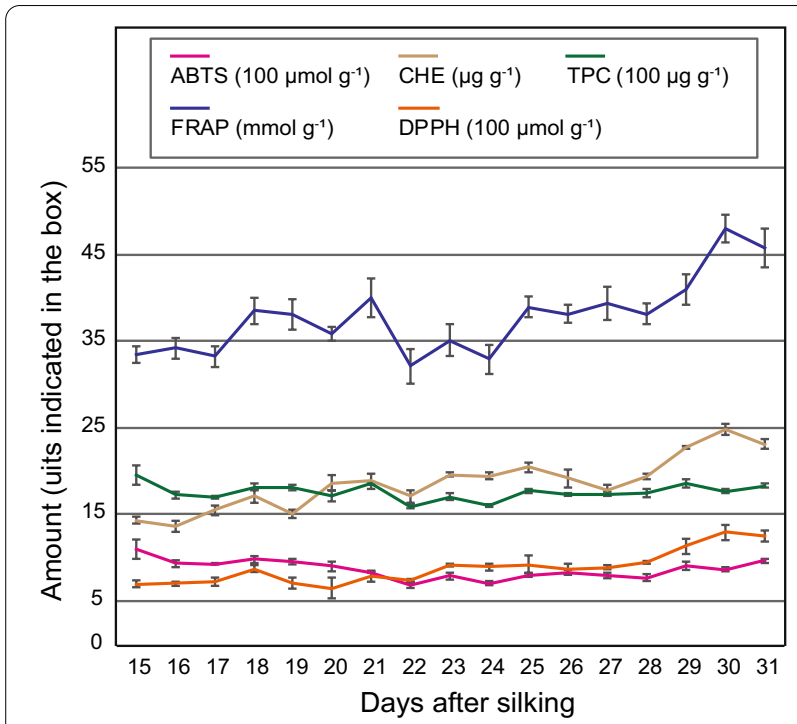

Fig. 3 Values for FRAP, ABTS, CHE, DPPH, and TPC during grain filling in purple corn 'Heukjinjuchal' kernels. Values are means from three biological replicates and error bars represent the SD of three biological replicates

anthocyanin content also increased at 27-30 DAS, but did not fluctuate at the earlier time points.

Although ABTS, DPPH, and FRAP are electron transfer-based assays, they produced different results in this study. $\mathrm{Xu}$ et al. [34] reported that antioxidant activity measured by DPPH, FRAP, and TPC decreased during grain filling and maturation in yellow corn kernels from their 12-day interval samples, whereas the increase in antioxidant activity in this study was likely due to anthocyanin accumulation.

\section{Comparisons among five antioxidant assays}

We next investigated correlations among the antioxidant levels as measured by the different assays to find an appropriate combination of methods for analyzing the antioxidant activity of fresh purple corn samples. The correlations among the measurements generated by the five different antioxidant assays and the anthocyanin content of purple corn kernels are shown in Table 1. The differences among the antioxidant levels often showed weak or no correlations. Nevertheless, highly significant correlations were identified between DPPH and FRAP, DPPH and CHE, FRAP and CHE, and ABTS and TPC levels $\left(r=0.828^{* * * *}, 0.893^{* * * *}, 0.761^{* * * *}\right.$, and $0.764^{* * * *}$, respectively; Table 1). The levels of DPPH, FRAP, and CHE were strongly correlated and showed similar patterns to that of anthocyanin accumulation.

Notably, significant correlations were observed among the antioxidants measured by the five assay methods after 22 DAS $\left(r=0.699^{*}-0.961^{* * * * ;}\right.$; Table 2$)$. The correlations
Table 1 Correlation analysis between FRAP, ABTS, CHE, DPPH, and TPC levels during grain filling (15-31 DAS) in purple corn kernels

\begin{tabular}{llrrlc}
\hline & FRAP & ABTS & CHE & TPC & Anthocyanins \\
\hline DPPH & $0.828^{* * *}$ & -0.081 & $0.893^{* * *}$ & 0.179 & $0.924^{* * *}$ \\
FRAP & & 0.153 & $0.761^{* * *}$ & 0.431 & $0.905^{* * *}$ \\
ABTS & & & -0.281 & $0.764^{* * *}$ & $0.798^{* * *}$ \\
CHE & & & & 0.043 & -0.246 \\
TPC & & & & & 0.005 \\
\hline
\end{tabular}

Numbers represent Pearson's $R$

***Represents significance at $p<0.001$

Table 2 Correlation analysis between FRAP, ABTS, CHE, DPPH, and TPC levels during late grain filling (22-31 DAS) in purple corn kernels

\begin{tabular}{llllll}
\hline & FRAP & ABTS & CHE & TPC & Anthocyanins \\
\hline DPPH & $0.961^{* * *}$ & $0.914^{* * *}$ & $0.830^{* *}$ & $0.729^{*}$ & $0.937^{* * *}$ \\
FRAP & & $0.849^{* *}$ & $0.783^{* *}$ & $0.699^{*}$ & $0.864^{* * *}$ \\
ABTS & & & $0.847^{* *}$ & $0.794^{* *}$ & $0.891^{* * *}$ \\
CHE & & & & $0.918^{* * *}$ & $0.728^{*}$ \\
TPC & & & & & 0.559
\end{tabular}

Numbers represent Pearson's $R$

${ }^{*}, * *$, and ${ }^{* * *}$ represents significance at $p<0.05, p<0.01$, and $p<0.001$, respectively

between DPPH and FRAP, DPPH and CHE, FRAP and CHE, and ABTS and TPC levels at 22-31 DAS were higher than those at 15-31 DAS. This suggests that corn kernels undergo huge physicochemical changes during early grain filling, which leads to inconsistencies among the levels of antioxidants measured.

Anthocyanin and phenols are two major antioxidant compounds present in purple corn kernels [14] and antioxidant activity has been shown to relate to phenolic content [35]. Moreover, corn generally shows higher TPC and antioxidant activity than other cereals, such as wheat, oats, and rice [1]. In our purple corn samples, the amount of phenolics remained stable during grain filling, whereas the concentration of anthocyanins continuously increased. The discrepancies among the methods seem to arise from the differences in these two main antioxidant compounds, anthocyanins and phenolics, which accumulate differentially during grain filling. According to the correlation analysis, the levels of DPPH, CHE, FRAP are more correlated with each other and those of ABTS and TPC are less-well correlated. The first three reflect anthocyanin content better than the latter two antioxidants.

Many researchers have reported the antioxidant properties of colored corn $[14,16,36]$. Pozo-Insfran et al. [37] demonstrated that red and blue maize had higher 
phenolic contents than light-colored maize genotypes. Although several studies have investigated the correlation between antioxidants assayed by different methods, no correlation between TPC and anthocyanin levels has been demonstrated [20,21, 26, 31]. The differences among the reported antioxidant assay results can be associated with the harvest time and types of plant samples, extraction solvents and methods, structures of the antioxidant compounds, and the ratios between the levels of these compounds [30]. $\mathrm{Hu}$ and $\mathrm{Xu}$ [14] reported that TPC and total anthocyanin content had different effects on the antioxidant activity of corn during grain maturation. In addition, it is difficult to evaluate the antioxidant activity of purple corn kernels during grain filling using only one assay, because the phytochemical composition of the sample varies greatly at different growth stages. Therefore, combinations of antioxidant activity assays are required to precisely estimate the antioxidant activities in purple corn kernels during grain filling. The combination of at least two assays, one from DPPH, CHE, and FRAP, and the other from ABTS and TPC are recommended for precise antioxidant activity measurement according to the correlations among the five assays we found in this study. Furthermore, additional studies with different genotypes are necessary to make conclusions concerning the use of such combinations.

\section{Supplementary Information}

The online version contains supplementary material available at https://doi. org/10.1186/s13765-021-00671-w.

Additional file 1: Table S1. FRAP, ABTS, CHE, DPPH, TPC, and anthocyanins levels during grain filling (15-31 DAS) in purple corn kernels.

\section{Authors' contributions}

Conceptualization: JTK, IMC, GY. Material preparation: JTK, IMC, MJK, JSL, BYS, HHB, YSG, SLK, SBB, SHK. Data acquisition and analysis: JTK, MJK, SHK. Data curation: JTK, GY. Writing original draft: JTK, GY. Review and editing: GY. All authors read and approved the final manuscript.

\section{Funding}

This work was carried out with the support of the 'Cooperative Research Program for Agriculture Science \& Technology Development in Rural Development Administration (Project No. PJ01249702)', Republic of Korea, and formed part of the Ph.D. thesis of Jung-Tae Kim, entitled 'Analysis of physicochemical characteristics, antioxidants, anthocyanins and proteome during the ripening stage on purple corn (Zea mays L.) 'Heukjinjuchal".

\section{Declarations}

\section{Competing interests}

The authors declare that they have no competing interests.

\section{Author details}

${ }^{1}$ R\&D Coordination Division, RDA, Jeonju 54875, Republic of Korea. ${ }^{2}$ Department of Crop Science, Sanghuh College of Life Sciences, Konkuk University, Seoul 05029, Republic of Korea. ${ }^{3}$ Department of Central Area Crop Science, National Institute of Crop Science, RDA, Suwon 16429, Republic of Korea.
${ }^{4}$ Department of Bio-Environmental Chemistry, College of Agriculture and Life Sciences, Chungnam National University, Daejoen 34134, Republic of Korea.

Received: 4 August 2021 Accepted: 27 December 2021

Published online: 06 January 2022

\section{References}

1. Adom KK, Liu RH (2002) Antioxidant activity of grains. J Agric Food Chem 50:6182-6187

2. Chatham LA, Paulsmeyer M, Juvik JA (2019) Prospects for economical natural colorants: insights from maize. Theor Appl Genet 132:2927-2946

3. Loarca-Piña G, Neri M, Figueroa JD, Castaño-Tostado E, Ramos-Gómez M, Reynoso R et al (2019) Chemical characterization, antioxidant and antimutagenic evaluations of pigmented corn. J Food Sci Technol 56:3177-3184

4. Chen Z, Zhang R, Shi W, Li L, Liu H, Liu Z et al (2019) The multifunctional benefits of naturally occurring delphinidin and its glycosides. J Agric Food Chem 67:11288-11306

5. Kähkönen MP, Heinonen M (2003) Antioxidant activity of anthocyanins and their aglycons. J Agric Food Chem 51:628-633

6. Tsuda T, Horio F, Osawa T (2002) Cyanidin 3-O- $\beta$-D-glucoside suppresses nitric oxide production during a zymosan treatment in rats. J Nutr Sci Vitaminol 48:305-310

7. Venancio VP, Cipriano PA, Kim H, Antunes LMG, Talcott ST, Mertens-Talcott SU (2017) Cocoplum (Chrysobalanus icaco L.) anthocyanins exert antiinflammatory activity in human colon cancer and non-malignant colon cells. Food Funct 8:307-314

8. Chen J, Zhu Y, Zhang W, Peng X, Zhou J, Li F et al (2018) Delphinidin induced protective autophagy via mTOR pathway suppression and AMPK pathway activation in HER-2 positive breast cancer cells. BMC Cancer 18:342

9. Hagiwara A, Miyashita K, Nakanishi T, Sano M, Tamano S, Kadota T et al (2001) Pronounced inhibition by a natural anthocyanin, purple corn color, of 2-amino-1-methyl-6-phenylimidazo [4, 5-b] pyridine (PhIP)-associated colorectal carcinogenesis in male F344 rats pretreated with 1, 2-dimethylhydrazine. Cancer Lett 171:17-25

10. Jeong MH, Ko H, Jeon H, Sung GJ, Park SY, Jun WJ et al (2016) Delphinidin induces apoptosis via cleaved HDAC3-mediated p53 acetylation and oligomerization in prostate cancer cells. Oncotarget 7:56767-56780

11. Fruebis J, Tsao T-S, Javorschi S, Ebbets-Reed D, Erickson MRS, Yen FT et al (2001) Proteolytic cleavage product of 30-kDa adipocyte complementrelated protein increases fatty acid oxidation in muscle and causes weight loss in mice. Proc Natl Acad Sci USA 98:2005-2010

12. Parra-Vargas M, Sandoval-Rodriguez A, Rodriguez-Echevarria R, Dominguez-Rosales JA, Santos-Garcia A, Armendariz-Borunda J (2018) Delphinidin ameliorates hepatic triglyceride accumulation in human HepG2 cells, but not in diet-induced obese mice. Nutrients 10:1060

13. Tsuda T, Ueno Y, Aoki H, Koda T, Horio F, Takahashi N et al (2004) Anthocyanin enhances adipocytokine secretion and adipocyte-specific gene expression in isolated rat adipocytes. Biochem Biophys Res Commun 316:149-157

14. Hu QP, Xu JG (2011) Profiles of carotenoids, anthocyanins, phenolics, and antioxidant activity of selected color waxy corn grains during maturation. J Agric Food Chem 59:2026-2033

15. Lim S, Yi G (2019) Investigating seed mineral composition in Korean landrace maize (Zea mays L.) and its kernel texture specificity. J Integr Agric 18:1996-2005

16. Lopez-Martinez LX, Oliart-Ros RM, Valerio-Alfaro G, Lee CH, Parkin KL, Garcia HS (2009) Antioxidant activity, phenolic compounds and anthocyanins content of eighteen strains of Mexican maize. LWT Food Sci Technol 42:1187-1192

17. Yang Z, Zhai W (2010) Identification and antioxidant activity of anthocyanins extracted from the seed and cob of purple corn (Zea mays L.). Innov Food Sci Emerg Technol 11:169-176

18. Bae HH, Yi G, Go YS, Ha JY, Choi YC, Son JH et al (2021) Measuring antioxidant activity in yellow corn (Zea mays L.) inbreds from three different geographic regions. Appl Biol Chem 64:56

19. Huang D, Ou B, Prior RL (2005) The chemistry behind antioxidant capacity assays. J Agric Food Chem 53:1841-1856 
20. Dudonné S, Vitrac X, Coutiere P, Woillez M, Mérillon JM (2009) Comparative study of antioxidant properties and total phenolic content of 30 plant extracts of industrial interest using DPPH, ABTS, FRAP, SOD, and ORAC assays. J Agric Food Chem 57:1768-1774

21. Frankel EN, Meyer AS (2000) The problems of using one-dimensional methods to evaluate multifunctional food and biological antioxidants. J Sci Food Agric 80:1925-1941

22. Kim JT, Yi G, Chung IM, Son BY, Bae HH, Go YS et al (2020) Timing and pattern of anthocyanin accumulation during grain filling in purple waxy corn (Zea mays L.) suggest optimal harvest dates. ACS Omega 5:15702-15708

23. Kim JT, Yi G, Kim MJ, Son BY, Bae HH, Go YS et al (2020) Glycolysis stimulation and storage protein accumulation are hallmarks of maize (Zea mays L.) grain filling. Appl Biol Chem 63:54

24. Jung TW, Song S, Son BY, Kim JT, Baek SB, Kim CK et al (2009) A black waxy hybrid corn, "Heukjinjuchal" with good eating quality. Korean J Breed Sci 41:599-602

25. Abdel-Aal E-SM, Hucl P (1999) A rapid method for quantifying total anthocyanins in blue aleurone and purple pericarp wheats. Cereal Chem 76:35-354

26. Kim JT, Chung IM, Son BY, Lee JS, Kim SL, Baek SB et al (2014) Comparison of the antioxidant activity of maysin (C-Glycosylflavone) and other flavonoids. Asian J Chem 26:2931-2934. https://doi.org/10.14233/ajchem. 2014.16074

27. Kim HB, Kim JB, Seok YS, Seo SD, Kim SL, Sung GB (2012) The effect of citric acid and enzyme inactivation treatment on C3G stability and antioxidant capacity of mulberry fruit jam. J Sericult Entomol Sci 50:82-86. https://doi.org/10.7852/jses.2012.50.2.82

28. Horwitz W (1980) Official methods of analysis of the Association of Official Analytical Chemists. AOAC, Washington, D.C

29. Pang Z, Chong J, Zhou G, de Lima Morais DA, Chang L, Barrette M et al (2021) MetaboAnalyst 5.0: narrowing the gap between raw spectra and functional insights. Nucleic Acids Res 49:W388-W396

30. Ž̈lić S, Serpen A, Akıllıoğlu G, Gökmen V, Vančetović J (2012) Phenolic compounds, carotenoids, anthocyanins, and antioxidant capacity of colored maize (Zea mays L.) kernels. J Agric Food Chem 60:1224-1231

31. Buenger J, Ackermann $H$, Jentzsch A, Mehling A, Pfitzner I, Reiffen KA et al (2006) An interlaboratory comparison of methods used to assess antioxidant potentials. Int J Cosmet Sci 28:135-146

32. Miller NJ, Rice-Evans C, Davies MJ, Gopinathan V, Milner A (1993) A novel method for measuring antioxidant capacity and its application to monitoring the antioxidant status in premature neonates. Clin Sci 84:407-412

33. Popov I, Lewin G (2008) Handbook of chemiluminescent methods in oxidative stress assessment. Research Signpost, Trivandrum

34. Xu JG, Hu QP, Wang XD, Luo JY, Liu Y, Tian CR (2010) Changes in the main nutrients, phytochemicals, and antioxidant activity in yellow corn grain during maturation. J Agric Food Chem 58:5751-5756

35. Wong CC, Li HB, Cheng KW, Chen F (2006) A systematic survey of antioxidant activity of 30 Chinese medicinal plants using the ferric reducing antioxidant power assay. Food Chem 97:705-711

36. González-Muñoz A, Quesille-Villalobos AM, Fuentealba C, Shetty K, Ranilla LG (2013) Potential of Chilean native corn (Zea mays L.) accessions as natural sources of phenolic antioxidants and in vitro bioactivity for hyperglycemia and hypertension management. J Agric Food Chem 61:10995-11007

37. Pozo-Insfran DD, Serna Saldivar SO, Brenes CH, Talcott ST (2007) Polyphenolics and antioxidant capacity of white and blue corns processed into tortillas and chips. Cereal Chem 84:162-168

\section{Publisher's Note}

Springer Nature remains neutral with regard to jurisdictional claims in published maps and institutional affiliations.

\section{Submit your manuscript to a SpringerOpen ${ }^{\circ}$ journal and benefit from:}

- Convenient online submission

- Rigorous peer review

- Open access: articles freely available online

- High visibility within the field

- Retaining the copyright to your article

Submit your next manuscript at $\boldsymbol{\nabla}$ springeropen.com 